\title{
An Ascending Vickrey Auction for Selling Bases of a Matroid*
}

\author{
Sushil Bikhchandani ${ }^{\dagger} \quad$ Sven de Vries ${ }^{\ddagger} \quad$ James Schummer $^{\S}$ \\ Rakesh V. Vohra
}

May 19, 2005

\begin{abstract}
We consider an ascending auction to sell the elements of a matroid. The value of each element is private information to the bidders. Bidding sincerely is an equilibrium of the auction and the elements sold form a maximum weight basis of the matroid. As a corollary we obtain the ascending auction by Ausubel (2004) for selling homogeneous goods with decreasing marginal values.
\end{abstract}

Keywords Vickrey auctions, matroids, multi-item auctions, combinatorial auctions,

${ }^{*}$ We gratefully acknowledge invaluable discussions with Joe Ostroy at U.C.L.A.

†Anderson Graduate School of Management, UCLA, Los Angeles, CA 90095.

‡Zentrum Mathematik, TU München, 85747 Garching bei München, Germany.

${ }^{\S}$ Department of Managerial Economics and Decision Sciences, Kellogg Graduate School of Management, Northwestern University, Evanston IL 60208.

IDepartment of Managerial Economics and Decision Sciences, Kellogg Graduate School of Management, Northwestern University, Evanston IL 60208. 


\section{Introduction}

This paper is concerned with the design of an ascending auction to sell off the elements of a matroid. The setting considered involves a finite ground set $E$ and a set $N$ of agents. Each agent $j \in N$ is associated with a set $E_{j} \subseteq E$ that $j$ is interested in acquiring. We assume without loss of generality that $\left(E_{j}\right)_{j \in N}$ is a partition of $E$ for otherwise joint elements could be replaced by parallel elements. Denote the value of each element $e \in E$ to the relevant agent by $v_{e} \in \mathbb{N}_{+}$. We assume this to be a positive integer and known only to the relevant agent. The value to agent $j$ of a set $S \subseteq E_{j}$ is $v(S):=\sum_{e \in S \cap E_{j}} v_{e}$.

We want the ascending auction to have two features. The first is that agents have an incentive to bid sincerely. Second, the set of elements sold should constitute a maximum weight basis. If the valuations of the elements of the matroid were known to the auctioneer the problem is trivial. In our case the valuations are private information.

The setting may seem abstract at first, but contains at least two economically relevant settings as a special case. One is the sale of multiple units of a homogenous good to agents with diminishing marginal utility. The ascending auction of Ausubel (2004) is a special case of the auction developed here. The second is the sale of a minimally connected network (spanning tree). Matroid techniques also play a role in the design of ascending auctions in other environments, see Gul and Stacchetti (1999, 2000).

A sealed bid auction with the two features we desire is available. It is the generalized Vickrey auction. Nevertheless, there are reasons for eschewing the sealed bid Vickrey auction. These are described, for example by Ausubel (2004), Cramton (1998). The goal of this paper is to describe an ascending auction that implements the outcome of the sealed bid Vickrey auction.

In the next section we describe the sealed bid Vickrey auction and prior work on desiging ascending auctions that implement the Vickrey auction. Subsequently we use a particular matroid (graphic) to motivate the main ideas. The remaining sections summarize the main facts of matroid theory that we use and the subsequent analysis.

\section{The Vickrey Auction}

Consider a set of of agents $N$, each of whom has a (monetary) value $v^{j}\left(a_{j}\right)$ for any bundle $a_{j}$ received under some "allocation" of goods $a \in A$. The 
seller's objective is to find the "efficient" allocation $a^{*}$ which solves $V(N):=$ $\max _{a \in A} \sum_{j \in N} v^{j}\left(a_{j}\right)$.

Consider a situation in which bidder $k \in N$ is absent. In this case the seller's objective is to find $V(N \backslash k):=\max _{a \in A} \sum_{j \in N \backslash k} v^{j}\left(a_{j}\right)$. Thus, the net effect that $k$ 's presence has on the other bidders equals

$$
V(N \backslash k)-\sum_{j \in N \backslash k} v^{j}\left(a_{j}^{*}\right)
$$

which is precisely bidder $k$ 's Vickrey payment. Bidder $k$ 's net payoff in a Vickrey auction is therefore

$$
v^{k}\left(a_{k}^{*}\right)-\left[V(N \backslash k)-\sum_{j \in N \backslash k} v^{j}\left(a_{j}^{*}\right)\right]=V(N)-V(N \backslash k) .
$$

That is, his net payoff equals his net contribution to attainable social surplus, which is why this amount is also called bidder $k$ 's marginal product.

The payments in a Vickrey sealed-bid auction can be found by solving $n+$ 1 optimization problems: one to find $V(N)$ (and $a^{*}$ ), and $n$ more to find each $V(N \backslash j)$. However, in many environments the problem of finding $V(N)$ can be formulated as a simple linear program. Furthermore, an agent's presence can be reflected in the choice of constraints (rows) or variables (columns) of the linear program. Therefore, it is tempting to think that his marginal product, might be encoded in the optimal dual variables of the linear program - these variables inform us of the effect of changing the right hand side of a constraint. Whenever such a connection exists, payments for sealed-bid Vickrey auctions could be computed with a single linear program (producing $V(N)$ and $a^{*}$ ) and its dual (producing marginal products). Since each $v^{j}\left(a_{j}^{*}\right)$ can be computed from the program, the payments follow immediately from (1).

A byproduct of this desireable connection between linear programming variables and Vickrey payoffs/payments is useful for designing ascending auctions. While the sealed-bid version of the Vickrey auction has the appeal of the properties discussed above, there may, in some environments, be practical reasons to prefer a dynamic, ascending implementation of this auction outcome. $^{1}$

In de Vries et al. (2004) the duality approach is used to derive a new ascending auction for the sale of heterogeneous goods that (under some necessary conditions) results in truthinducing Vickrey payments. The authors provide a fully combinatorial version of that auction.

\footnotetext{
${ }^{1}$ Such reasons may include the auctioneer's credibility, perceptions of fairness, etc; see Ausubel (2004), Cramton (1998).
} 
Here we consider a matroid $\mathcal{M}=(E, \mathcal{I})$ with rank function $r$. The optimization problem we consider is that of finding a maximum weight basis of $\mathcal{M}$. We will assume, that the no monopoly condition holds, that requires that $r(\mathcal{M})=r\left(\mathcal{M}-E_{j}\right)$ for all $j \in N$. Notice that the no monopoly condition is fulfilled, if no cocircuit $C^{\star}$ of $\mathcal{M}$ belongs to any bidder (that is, $C^{\star}$ is not contained in any $E_{j}$ ).

We present a combinatorial algorithm for this problem which will implement the Vickrey outcome. But before proceeding, it is useful to consider a special case in order to develop some intuition.

\section{Example: Selling a Tree from a Graph}

Let $G=(V, E)$ be a complete graph with vertex set $V$ and edge set $E$. Each edge may be owned by a given agent and assume (for the sake of easier exposition) that an agent has the right to own only a single predetermined edge. Therefore we may use the words edge and agent interchangeably. Let $v_{e}$ be the value of edge $e$. Our goal is to derive an ascending Vickrey auction to sell off a maximum weight spanning tree. Notice, as we assume $G$ to be complete, no one agent is a in a position to hold up the auctioneer.

Though we shall speak in terms of "selling" edges, one interpretation for this problem involves a procurement setting, where the auctioneer wants to purchase the right to use an edge and the bidder incurs some cost $\left(-v_{e}\right)$ when it is used (e.g. constructing a complete communications network at minimal total social cost). In order to be consistent with the rest of the paper, we avoid procurement examples and say that the auctioneer is selling to bidders the right to use an edge, incurring a gain of $v_{e} \geq 0 .^{2}$

An important observation to make is that, instead of selling an edge, the auctioneer is actually selling the right to "cover" a cut in the graph. (Notice that cuts in graphs are cocircuits of the underlying graphical matroid.) A bidder is competing with all other bidders that can cover the same collection of cuts that he can. This can be seen when we compute the marginal product of an edge.

\footnotetext{
${ }^{2}$ If instead bidders incur costs $\left(v_{e}<0\right)$, then we can suppose that bidders bid on the right to supply their edges for some fixed payment $M$. If $M$ can be chosen sufficiently high to guarantee $M>v_{e}$ for each $e$, then, as all matroid bases have the same cardinality, this setting is equivalent to the one we describe.
} 
Let $T$ be a maximum weight spanning tree and suppose $e \in T$. To determine agent $e$ 's marginal product we must identify the reduction in weight of the spanning tree when we remove agent $e$ and replace her with a (next best) edge. If $f \notin T$ is the largest weight edge such that $T \cup f$ contains a cycle through $e$, then the maximum weight spanning tree that excludes $e$ is $(T \backslash\{e\}) \cup f$. Thus agent $e$ 's marginal product is $v_{e}-v_{f}$.

There are a number of algorithms for finding a maximum weight spanning tree, but not all lend themselves to an auction interpretation. Furthermore, not all of them terminate in Vickrey prices. The "greedy out" algorithm does: starting with the complete set of edges, delete edges in order of increasing weight. An edge is deleted only if the remaining graph is connected. An edge is spared from deletion when all smaller weight edges that could cover the same cut have already been deleted.

This algorithm can be interpreted as an auction which begins with a price $p:=0$ on each edge. Throughout the auction, this price is increased. At each point in time, each agent announces whether he is willing to purchase his edge at the current price.

As the price increases, agents drop out of the auction when the price exceeds their value $v_{e}$ for the edge, reducing the connectivity of the graph. At some point, an agent will become critical: removing the agent from the auction would mean that no spanning tree could be formed from the remaining edges of the other agents. At this point, the auctioneer immediately sells the edge to the critical agent at the current price. This edge is to be part of the final (maximum weight) spanning tree and does not drop out.

The auction then continues, with other agents dropping out or becoming critical. The auction ends when the last critical agent is awarded an edge, and the tree is formed.

Notice that a critical agent acquires his edge at the price where another bidder dropped out of the auction. That price is the second-largest weighted edge that could have covered the same cut as the critical agent. This is the price a Vickrey auction dictates he should pay.

In what follows we will present a combinatorial algorithm for this auction. The analysis will involve some fine points that arise because we will allow agents to have an interest in multiple elements of the matroid. 
RCS: $1.43,2005 / 05 / 19$ 01:10:53

\section{A Direct Algorithm for Computing the Vick- rey Outcome for Matroids}

We describe below Algorithm 1 due to Dawson (1980) which finds an optimum basis for a matroid.

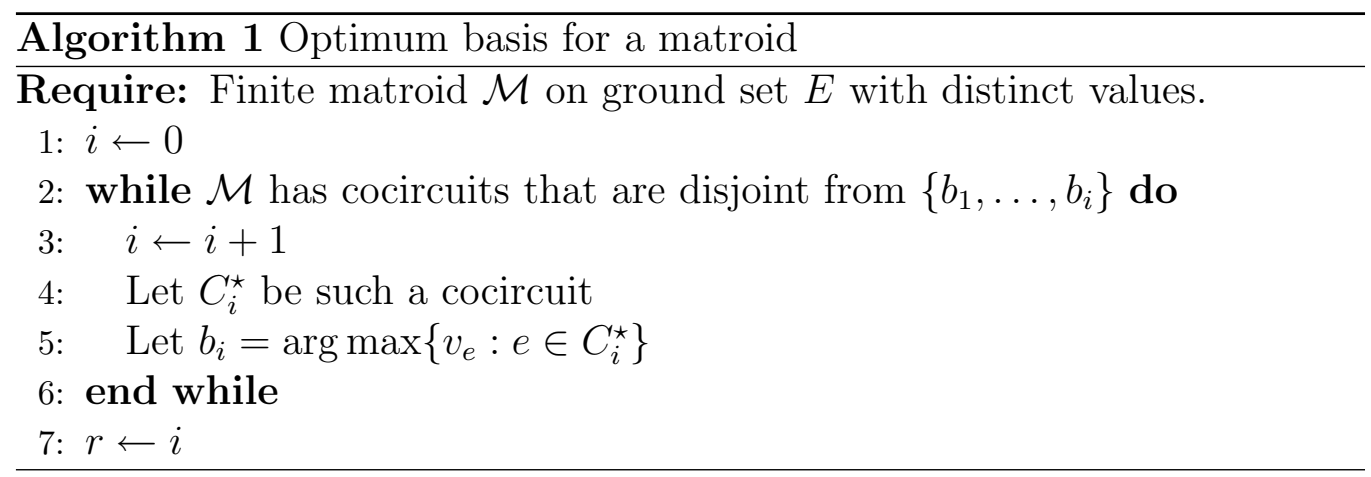

Proposition 1 (Dawson, 1980, Thm. 1). The set $\left\{b_{1}, \ldots, b_{r}\right\}$, returned by an application of Algorithm 1 to a matroid $\mathcal{M}$ with distinct values is an optimal basis of $\mathcal{M}$. (Consequently, the rank of $\mathcal{M}$ is r.)

The choice of the cocircuit $C_{i}^{\star}$ in Line 4 of the Algorithm 1 is arbitrary. Motivated by this algorithm, our Algorithm 2 below chooses cocircuits in a particular way to generate Vickrey prices.

A sequence of cocircuit-element pairs, $\left(\left(C_{1}^{\star}, b_{1}\right),\left(C_{2}^{\star}, b_{2}\right), \ldots,\left(C_{i}^{\star}, b_{i}\right)\right)$ that is constructed during the execution of Algorithm 1 will be called suitable. Therefore, a sequence $\left(\left(C_{1}^{\star}, b_{1}\right),\left(C_{2}^{\star}, b_{2}\right), \ldots,\left(C_{i}^{\star}, b_{i}\right)\right)$ is suitable for $\mathcal{M}$ iff $C_{j}^{\star}$ is a cocircuit, $b_{j}=\arg \max _{e \in C_{j}^{\star}} v_{e}$, and $C_{j}^{\star} \cap\left\{b_{1}, b_{2}, \ldots, b_{j-1}\right\}=\emptyset$ for $1 \leq j \leq i$. Observe that a suitable sequence of $r(\mathcal{M})$ cocircuits provides a certificate of independence and optimality for $\left\{b_{1}, \ldots, b_{r(\mathcal{M})}\right\}$.

First assume that the valuations on each element are distinct positive rationals. Thus a maximum weight basis of $\mathcal{M}$ (or of any of its minors) will be unique. Recall that we have a partition $E_{1}, \ldots, E_{n}$ of $E$, reflecting the 'ownership' of the elements, and assume the no-monopoly condition which ensures that $r(\mathcal{M})=r\left(\mathcal{M} \backslash E_{i}\right)$ for all $i$. Let $o(e)$ denote the index of the owner of the $E_{i}$ from the partition $E_{1}, \ldots, E_{n}$ that contains $e$.

For any cocircuit $C^{\star}$ of $\mathcal{M}$, let $b_{C^{\star}}=\arg \max \left\{v_{e}: e \in C^{\star}\right\}$ be its best element and $f_{C^{\star}}=\arg \max \left\{v_{e}: e \in C^{\star} \backslash E_{o\left(b_{C^{\star}}\right)}\right\}$ the best element of $C^{\star} \backslash$ 
$E_{o\left(b_{C^{\star}}\right)}$, i.e., the second best element of $C^{\star}$ associated with a bidder distinct from $o\left(b_{C^{\star}}\right)$. Since the no-monopoly condition holds, $C^{\star} \backslash E_{o\left(b_{C^{\star}}\right)} \neq \emptyset$ (if not, $C^{\star} \subseteq E_{o\left(b_{C^{\star}}\right)}$ and $\left.r\left(\mathcal{M} \backslash E_{o\left(b_{C^{\star}}\right)}\right) \leq r\left(\mathcal{M} \backslash C^{\star}\right)=r(\mathcal{M})-1\right)$. Hence $f_{C^{\star}}$ is well defined.

Call a cocircuit $C^{\star}$ of $\mathcal{M}$ feasible at $p \geq 0$ if $v_{f_{C^{\star}}}=p$. For each $e \in E$ we will say that the cocircuit $C^{\star}$ of $\mathcal{M}$ feasible at $p \geq 0$ for $e$ if $e=f_{C^{\star}}$ and $v_{f_{C^{\star}}}=p$.

The idea of Algorithm 2 is to select cocircuits $C^{\star}$ by non-decreasing value of $v_{f_{C^{\star}}}$. The resulting set of elements $B:=\left\{b_{1}, \ldots, b_{r}\right\}$ forms the optimum basis. The element $f_{C^{\star}}$ will turn out to be a "best" alternative to $b_{C^{\star}}$, if $E_{o\left(b_{C^{*}}\right)}$ were removed. First we describe the algorithm, then we show how to derive the Vickrey prices from its output.

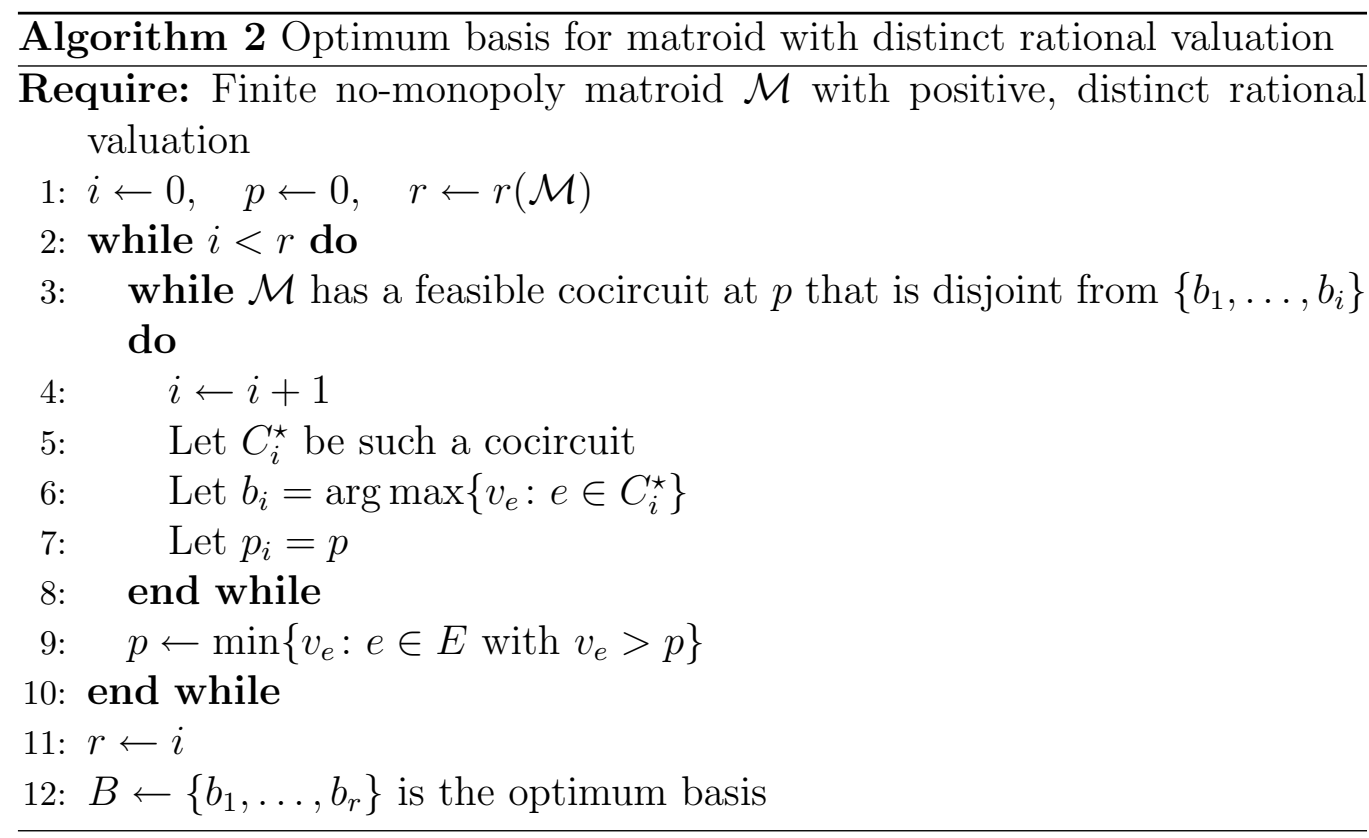

Algorithm 2 is a particular realization of Algorithm 1; hence the resulting sequence $\left(\left(C_{1}^{\star}, b_{1}\right),\left(C_{2}^{\star}, b_{2}\right), \ldots,\left(C_{r}^{\star}, b_{r}\right)\right)$ is suitable. The algorithm considers all cocircuits that avoid previously chosen elements because every cocircuit $C^{\star}$ is feasible with respect to one of the possible values that $p$ takes on. This implies the next theorem.

Theorem 2. The set $B=\left\{b_{1}, \ldots, b_{r}\right\}$ returned by Algorithm 2 applied to a finite, no-monopoly matroid $\mathcal{M}$ with positive, distinct rational valuations is the unique maximum weight basis. 
We show that the element $f_{C^{\star}}$ can be "second best" for at most one cocircuit per bidder.

Lemma 3. If the sequence determined by Algorithm 2 contains two cocircuits $C_{i}^{\star}, C_{j}^{\star}$ with o $\left(b_{i}\right)=o\left(b_{j}\right)$ then $f_{C_{i}^{\star}} \neq f_{C_{j}^{\star}}$.

Note that if $p_{i}<p_{j}$ then Lemma 3 follows from the definition of $f_{c}$ and the fact that $C_{i}^{\star}$ and $C_{j}^{\star}$ are feasible at different prices. Otherwise is a simple consequence of the next lemma.

Lemma 4. If the sequence determined by Algorithm 2 contains two distinct cocircuits $C_{i}^{\star}, C_{j}^{\star}$ that are feasible at the same $p \geq 0$, then $o\left(b_{i}\right) \neq o\left(b_{j}\right)$.

Proof. Suppose, for a contradiction that $f_{C_{i}^{\star}}=f_{C_{j}^{\star}}$ and $o\left(b_{i}\right)=o\left(b_{j}\right)$. Without loss of generality we may suppose that and $v_{b_{i}}>v_{b_{j}}$ and $o\left(b_{j}\right)=1$.

By strong circuit elimination, there exists a cocircuit $C^{\star} \subseteq\left(C_{i}^{\star} \cup C_{j}^{\star}\right)-f_{C_{i}^{\star}}$ that contains $b_{i}$. As $b_{i}$ is the highest valued element of $C_{i}^{\star} \cup C_{j}^{\star}$ it follows $\arg \max _{e \in C^{\star}} v_{e}=b_{i}$.

By construction, for all $e \in C_{i}^{\star} \backslash\left(E_{1}+f_{C_{i}^{\star}}\right)$ we have $v_{e}<v_{f_{C_{i}^{\star}}}=p$ and the same for $C_{j}^{\star}$ (here we use the fact that all values $v_{e}$ are distinct). As $f_{C_{i}^{\star}} \notin C^{\star}$ and $\arg \max _{e \in C_{i}^{\star} \backslash E_{1}} v_{e}=f_{C_{i}^{\star}}=\arg \max _{e \in C_{j}^{\star} \backslash E_{1}} v_{e}$ it follows that for all $e \in C^{\star} \backslash E_{1}$ that $v_{e}<v_{f_{C_{i}^{*}}}$. Further, since $C^{\star} \subseteq\left(C_{i}^{\star} \cup C_{j}^{\star}\right)$, no $b_{k}$ for $k<\min (i, j)$ belongs to $C^{\star}$. Therefore, the cocircuit $C^{\star}$ was feasible at price $\max \left\{v_{e}: e \in C^{\star} \backslash E_{1}\right\}<p$, and was chosen as $\left(C_{l}^{\star}, b_{l}\right)$ earlier for $l<\min (i, j)$. But then $b_{l} \in C_{l}^{\star}=C^{\star} \subseteq C_{i}^{\star} \cup C_{j}^{\star}$. Hence at least one of $C_{i}^{\star}$ or $C_{j}^{\star}$ contains $b_{l}$ and must be infeasible, a contradiction.

Lemma 5. For all $i, j, b_{i} \neq f_{C_{j}^{\star}}$ from the sequence determined by Algorithm 2.

Proof. The lemma is true for $i<j$. To see why, suppose not, i.e., $b_{i}=f_{C_{j}^{\star}} \in$ $C_{j}^{\star}$. Since $f_{C_{j}^{\star}} \in C_{j}^{\star}$ but it follows that $C_{j}^{\star}$ is not feasible.

For $i=j$ the lemma follows from the fact that $f_{C_{j}^{\star}}$ belongs to $E_{k}$ where $k \neq o\left(b_{j}\right)$.

For $i>j$ notice that $v_{f_{C_{i}^{\star}}}<v_{b_{i}}$. Since $v_{b_{i}}=v_{f_{C_{j}^{\star}}}$ it follows that $v_{f_{C_{i}^{\star}}}<$ $v_{f_{C_{j}^{\star}}}$ and that $i$ was feasible before $j$, implying $i<j$ in contradiction to the assumption. 
To relate a basis $B=\left\{b_{1}, \ldots, b_{r}\right\}$ found by Algorithm 2 to the Vickrey prices, we have to determine the optimal bases for each $\mathcal{M} \backslash E_{j}$. We will show that $B^{-j}:=\left(B \backslash E_{j}\right) \cup\left\{f_{C_{i}^{\star}}: b_{i} \in B \cap E_{j}\right\}$ is an optimal basis of $\mathcal{M} \backslash E_{j}$; this will be used to prove that the $p_{i}$ determined in Algorithm 2 are Vickrey payments.

Let the sequence $\mathcal{K}=\left(\left(C_{1}^{\star}, b_{1}\right),\left(C_{2}^{\star}, b_{2}\right), \ldots,\left(C_{r}^{\star}, b_{r}\right)\right)$ be given. Consider a fixed set $E_{j}$, without loss of generality $E_{1}$, and the sequence $\left\{b_{1}^{\prime}, \ldots, b_{r}^{\prime}\right\}$ defined by:

$$
b_{i}^{\prime}= \begin{cases}b_{i} & : \text { if } o\left(b_{i}\right) \neq 1 \\ f_{C_{i}^{\star}} & : \text { if } o\left(b_{i}\right)=1 .\end{cases}
$$

If we can find cocircuits that make the sequence $\left\{b_{1}^{\prime}, \ldots, b_{r}^{\prime}\right\}=B^{-1}$ suitable for $\mathcal{M} \backslash E_{1}$, then $B^{-1}$ is an optimal basis of $\mathcal{M} \backslash E_{1}$. Let $\mathcal{M}^{\prime}:=\mathcal{M} \backslash E_{1}$. Lemma 3 implies that any $f_{C_{i}^{\star}}$ belongs to at most one chosen cocircuit $C^{\star}$ of the suitable sequence $\mathcal{K}$ with $o\left(e_{C^{\star}}\right)=1$. Furthemore, the $f_{C_{i}^{\star}}$ for $i$ such that $o\left(b_{i}\right)=1$ are all distinct. By Lemma 5 no $f_{C_{i}^{\star}}$ equals any $b_{j}$ for $i, j$. Therefore all $b_{i}^{\prime}$ are different.

As $\mathcal{M}^{\prime}$ is a deletion minor of $\mathcal{M}$, it follows from Corollary 24 that for every cocircuit $C^{\star}$ of $\mathcal{M}$, the set $C^{\star} \backslash E_{1}$ is the union of cocircuits of $\mathcal{M}^{\prime}$. Let $C^{\prime \star}{ }_{i} \subseteq C_{i}^{\star}$, for each $i=1, \ldots, r$, be the cocircuit of $\mathcal{M}^{\prime}$ that contains $b_{i}^{\prime}$. The sequence $\left(\left(C_{1}^{\prime \star}, b_{1}^{\prime}\right),\left(C_{2}^{\prime \star}, b_{2}^{\prime}\right), \ldots,\left(C_{r}^{\prime \star}, b_{r}^{\prime}\right)\right)$ has the property that $b_{i}^{\prime} \in C_{i}^{\prime \star}$ and $b_{i}^{\prime}=\arg \max _{e \in C^{\prime \star}{ }_{i}} v_{e}$ by construction. As all $b_{i}^{\prime}$ are distinct, the $C^{\prime \star}{ }_{i}$ are also distinct. However, this sequence need not be suitable, as there could be indices $i<j$ with $b_{i}^{\prime}=f_{C_{i}^{\prime \star}} \in C_{j}^{\prime \star}$.

Theorem 6. Suppose a sequence

$$
\mathcal{K}:=\left(\left(C_{1}^{\prime \star}, b_{1}^{\prime}\right),\left(C_{2}^{\prime \star}, b_{2}^{\prime}\right), \ldots,\left(C_{r}^{\prime \star}, b_{r}^{\prime}\right)\right)
$$

of $\mathcal{M}^{\prime}$ such that

(1) all $b_{i}^{\prime}$ are different,

(2) $b_{i}^{\prime}=\arg \max _{e \in C^{\prime \star}} v_{e}$ for $1 \leq i \leq r$,

(3) and the sequence $\left(\left(C_{1}^{\prime \star}, b_{1}^{\prime}\right),\left(C_{2}^{\prime \star}, b_{2}^{\prime}\right) \ldots,\left(C^{\prime \star}, b_{j}^{\prime}\right)\right)$ is for some $j$ between 1 and $r$ suitable.

Then the cocircuit ${C^{\prime *}}^{\star * 1}$ can be modified so that conditions (1)-(3) hold for $j+1$.

Proof. If $\left(\left(C_{1}^{\prime \star}, b_{1}^{\prime}\right),\left(C_{2}^{\prime \star}, b_{2}^{\prime}\right) \ldots,\left(C_{j+1}^{\prime \star}, b_{j+1}^{\prime}\right)\right)$ is suitable we are done.

Suppose not and consider the smallest $i<j+1$ with $b_{i}^{\prime} \in C^{\prime \star}{ }_{j+1}$. Using strong circuit elimination we can choose a cocircuit $C^{\star}$ of $\mathcal{M}^{\prime}$ in $\left(C^{\prime \star}{ }_{i} \cup\right.$ 
$\left.C^{\prime \star}{ }_{j+1}\right)-b_{i}^{\prime}$ that contains $b_{j+1}^{\prime}$. We replace $C_{j+1}^{\prime \star}$ by $C^{\star}$. By assumption, $b_{k}^{\prime} \notin$ $\left(C_{i}^{\prime \star} \cup C^{\prime \star}{ }_{j+1}^{\star}\right)-b_{i}^{\prime}$ for every $k<i$. Therefore, $\left\{b_{1}^{\prime}, \ldots, b_{i}^{\prime}\right\} \cap C^{\star}=\emptyset$, and we can replace $C^{\prime \star}{ }_{j+1}$ by $C^{\star}$. Let

$$
\mathcal{K}^{\prime}=\left(\left(C_{1}^{\prime \star}, b_{1}^{\prime}\right),\left(C_{2}^{\prime \star}, b_{2}^{\prime}\right), \ldots,\left(C_{j}^{\prime \star}, b_{j}^{\prime}\right),\left(C^{\star}, b_{j+1}^{\prime}\right),\left(C_{j+2}^{\prime \star}, b_{j+2}^{\prime}\right), \ldots,\left(C_{r}^{\prime \star}, b_{r}^{\prime}\right)\right) .
$$

Now $\mathcal{K}^{\prime}$ fulfills (1)-(3) for $j$ and $\left\{b_{1}^{\prime}, \ldots, b_{i}^{\prime}\right\} \cap{C^{\prime}}_{j+1}^{\star}=\emptyset$. Either $\mathcal{K}^{\prime}$ fulfills (3) for $j+1$, or there exists another index $i^{\prime}>i$ with $b_{i^{\prime}}^{\prime} \in C^{\prime \star}{ }_{j+1}$, in which case we repeat the procedure until $\mathcal{K}^{\prime}$ fulfills (1)-(3) for $j+1$.

The hypotheses of the preceding theorem are satisfied by the output of Algorithm 2 and $j=1$. Repeated application of the theorem proves that there are cocircuits $C_{1}^{\prime \star}, C_{2}^{\prime \star}, \ldots, C_{r}^{\prime \star}$ that make the sequence

$$
\left(\left(C_{1}^{\prime \star}, b_{1}^{\prime}\right),\left(C_{2}^{\prime \star}, b_{2}^{\prime}\right), \ldots,\left(C_{r}^{\prime \star}, b_{r}^{\prime}\right)\right)
$$

suitable in $\mathcal{M} \backslash E_{i}$; therefore $B^{-1}$ is optimal for $\mathcal{M} \backslash E_{1}$ (and analogously $B^{-i}$ is an optimal basis of $\mathcal{M} \backslash E_{i}$ for all $\left.i \in N\right)$.

Denote by $V(\mathcal{N})$ the value of a maximum weight basis in $\mathcal{N}$. The following summarizes what has been established so far.

Theorem 7. Suppose Algorithm 2 applied to a finite, no-monopoly matroid $\mathcal{M}$ with positive, distinct rational valuation returns the set $B=\left\{b_{1}, \ldots, b_{r}\right\}$. Then, $B$ is the unique maximum weight basis, the sets $B^{-i}$ are unique maximum weight bases for $\mathcal{M} \backslash E_{i}$ for $i \in N$. Bidder $i$ 's Vickrey surplus (defined as $\left.V(\mathcal{M})-V\left(\mathcal{M} \backslash E_{i}\right)\right)$ is $\sum_{j: b_{j} \in\left\{b_{1}, \ldots, b_{r}\right\} \cap E_{i}}\left(v_{b_{j}}-v_{f_{C_{j}^{\star}}}\right)$, and his Vickrey payment is $\sum_{j: b_{j} \in\left\{b_{1}, \ldots, b_{r}\right\} \cap E_{i}} v_{f_{C_{j}^{\star}}}=\sum_{j: b_{j} \in\left\{b_{1}, \ldots, b_{r}\right\} \cap E_{i}} p_{j}$.

The last identity follows from the observation that at price $p_{j}$ the cocircuit $C_{j}^{\star}$ is feasible, implying that $p_{j}=v_{f_{C_{j}^{\star}}}$.

The theorem shows that if bidder $i$ is awarded element $b_{j}$, then his final payment increases by $p_{j}$. Therefore, he can be charged $p_{j}$ at the time he is awarded element $b_{j}$. We use this observation to give an auction version of Algorithm 2 in Algorithm 3. From the previous theorem it follows that Algorithm 3 computes the optimal basis and charges Vickrey prices.

We show that elements with value less than $p$ are irrelevant during the computations for $p^{\prime}>p$ in Algorithm 2:

Lemma 8. In steps 4 and 6 of Algorithm 3, the computation can be performed for any $i>0$ in $\mathcal{M} \backslash\left\{e \in E \backslash\left\{b_{1}, \ldots, b_{i}\right\}: v_{e}<p_{i}\right\}$ without changing the result. 


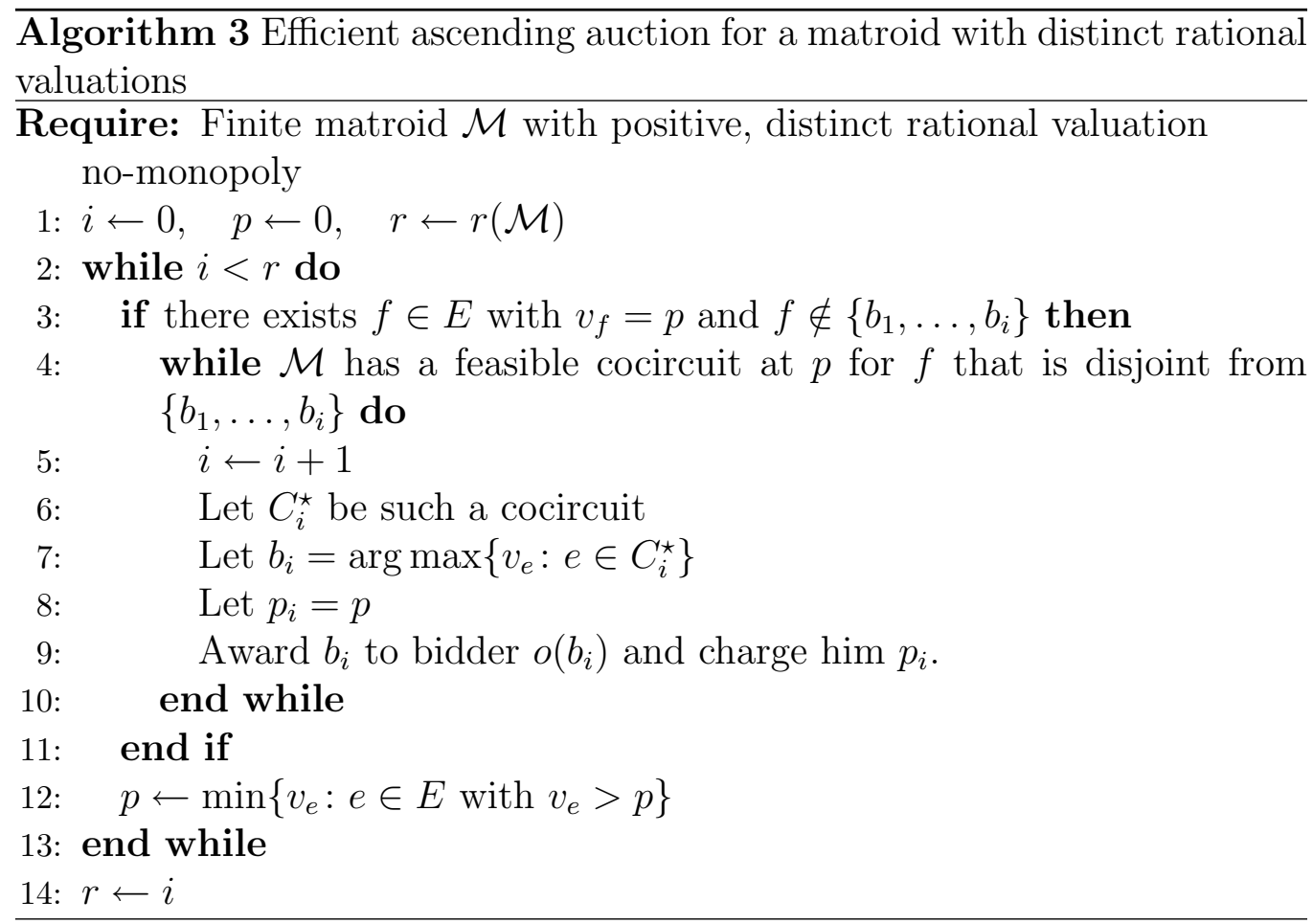

Proof. The lemma is true for $p_{1}=0$. Suppose it is true for some $p_{i}$. We show that it holds for $p_{i+1}$. If $p_{i}=p_{i+1}$ there is nothing prove; so consider the case where $p_{i}$ is increased in Line 9 to $p^{\prime}=\min \left\{v_{e}: e \in E\right.$ with $\left.v_{e}>p_{i}\right\}$ and where $i$ is later increased in Line 4 so that $p_{i+1}=p^{\prime}$ and $f$ is deleted.

There will not be another feasible cocircuit for $f$ in a later step, as all feasible cocircuits for $f$ are considered during the while-loop 3-8.

Similarily, $f$ cannot be the most valuable element of some feasible cocircuit at some $p^{\prime \prime}>p_{i}$, since the most valuable element of a feasible cocircuit for $p^{\prime \prime}$ has to have value greater than $p^{\prime \prime}$. Hence, omitting the element $f \in E \backslash\left\{b_{1}, \ldots, b_{i}\right\}$ with $v_{f}=p_{i}$ (if there is any) does not influence the output of the algorithm. As $f$ is the only new element with $v_{f}<p^{\prime}$, and all nonselected elements $e$ with $v_{e}<p$ have been previously removed, the lemma follows. ${ }^{3}$

When valuations can be arbitrary positive integers there may be two

\footnotetext{
${ }^{3}$ Notice that the matroids of type $\mathcal{M} \backslash\left\{e \in E \backslash\left\{b_{1}, \ldots, b_{i}\right\}: v_{e}<p_{i}\right\}$ usually violate the no-monopoly condition.
} 
different elements $e, f \in E$ with $v_{e} \leq v_{f}$ and $v_{f} \leq v_{e}$. We refer to this situation as a tie. Below we describe how to handle such ties.

\subsection{Tie Breaking}

We will break ties with the following (standard) device; let $0<\delta<1 / 4$ and suppose $E$ is a set of positive integers. For each $e \in E$ set $\epsilon_{e}=\delta^{e}$. Now we use the perturbed valuation $v^{\prime}(S):=v(S)+\sum_{e \in S} \epsilon_{e}$. The perturbed valuation has the property that if $v^{\prime}(S) \leq v^{\prime}(T)$ and $v$ is an integral valuation, then $v(S) \leq v(T)$, as $\delta$ is sufficiently small. (Clearly, for rational valuations the same could be done with a symbolic perturbation, without assigning a value to $\delta$ but only assuming it being small enough.)

Denote the value of a maximum basis of the matroid $\mathcal{N}$ with respect to the valuation $v^{\prime}$ by $V^{\prime}(\mathcal{N})$. The next result relates the maximum weight basis with respect to $v^{\prime}$ to the maximum weight basis with respect to $v$.

Lemma 9. Let $B \subseteq E$ fulfill $v^{\prime}(B)=V^{\prime}(\mathcal{M})$ and $B^{-i} \subseteq E$ fulfill $v^{\prime}\left(B^{-i}\right)=$ $V^{\prime}\left(\mathcal{M} \backslash E^{i}\right)$ then $v(B)=V(\mathcal{M})$ and $v\left(B^{-i}\right)=V\left(\mathcal{M} \backslash E^{i}\right)$ for $i \in N$.

Proof. Consider the elements of $\mathcal{M}$ ordered by nonincreasing $v^{\prime}$ value. Clearly, they are nonincreasing by value $v$. Therefore an application of the usual greedy-algorithm on this ordering which returns $B$ for $v^{\prime}$ returns $B$ also for $v$. The proof for the set $B^{-i}$ is analogous.

We generalize Theorem 7

Lemma 10. Let $B=\left\{b_{1}, \ldots, b_{r}\right\}$ be the set returned by Algorithm 3 when applied to the perturbed valuation $v^{\prime}$ of a finite, no-monopoly matroid $\mathcal{M}$ with positive integer valuation $v$. Then $B$ is the unique maximum weight basis with respect to $v^{\prime}$. The sets $B^{-i}$ are the unique maximum weight bases with respect to $v^{\prime}$ for $\mathcal{M} \backslash E_{i}$ for $1 \leq i \leq n$. As a consequence,

1. bidder $i$ 's marginal product with respect to $v^{\prime}$ is $\sum_{j: b_{j} \in\left\{b_{1}, \ldots, b_{r}\right\} \cap E_{i}}\left(v_{b_{j}}^{\prime}-\right.$ $\left.v_{f_{C_{j}^{\star}}}^{\prime}\right)$, hence his Vickrey payment is $\sum_{j: b_{j} \in\left\{b_{1}, \ldots, b_{r}\right\} \cap E_{i}} v_{f_{C_{j}^{\star}}^{\prime}}$.

2. bidder $i$ 's marginal product with respect to $v$ is $\sum_{j: b_{j} \in\left\{b_{1}, \ldots, b_{r}\right\} \cap E_{i}}\left(v_{b_{j}}-\right.$ $v_{f_{C_{j}^{\star}}}$, hence his Vickrey payment is $\sum_{j: b_{j} \in\left\{b_{1}, \ldots, b_{r}\right\} \cap E_{i}} v_{f_{C_{j}^{*}}}=\sum_{j: b_{j} \in\left\{b_{1}, \ldots, b_{r}\right\} \cap E_{i}} p_{j}$.

Proof. The first part is a direct consequence of Theorem 7 applied to the valuation $v^{\prime}$. From the previous lemma, we know that the unique optimal solutions to $V^{\prime}(\mathcal{M})$ and $V^{\prime}\left(\mathcal{M} \backslash E_{i}\right)$ are optimal for $V(\mathcal{M})$ and $V\left(\mathcal{M} \backslash E_{i}\right)$, too, which implies the second part. 
RCS: 1.43, 2005/05/19 01:10:53

In Algorithm 4 we modify Algorithm 3 so that it uses the perturbed order $v^{\prime}$ for tie breaking but charges prices that are consistent with the original valuation $v$.

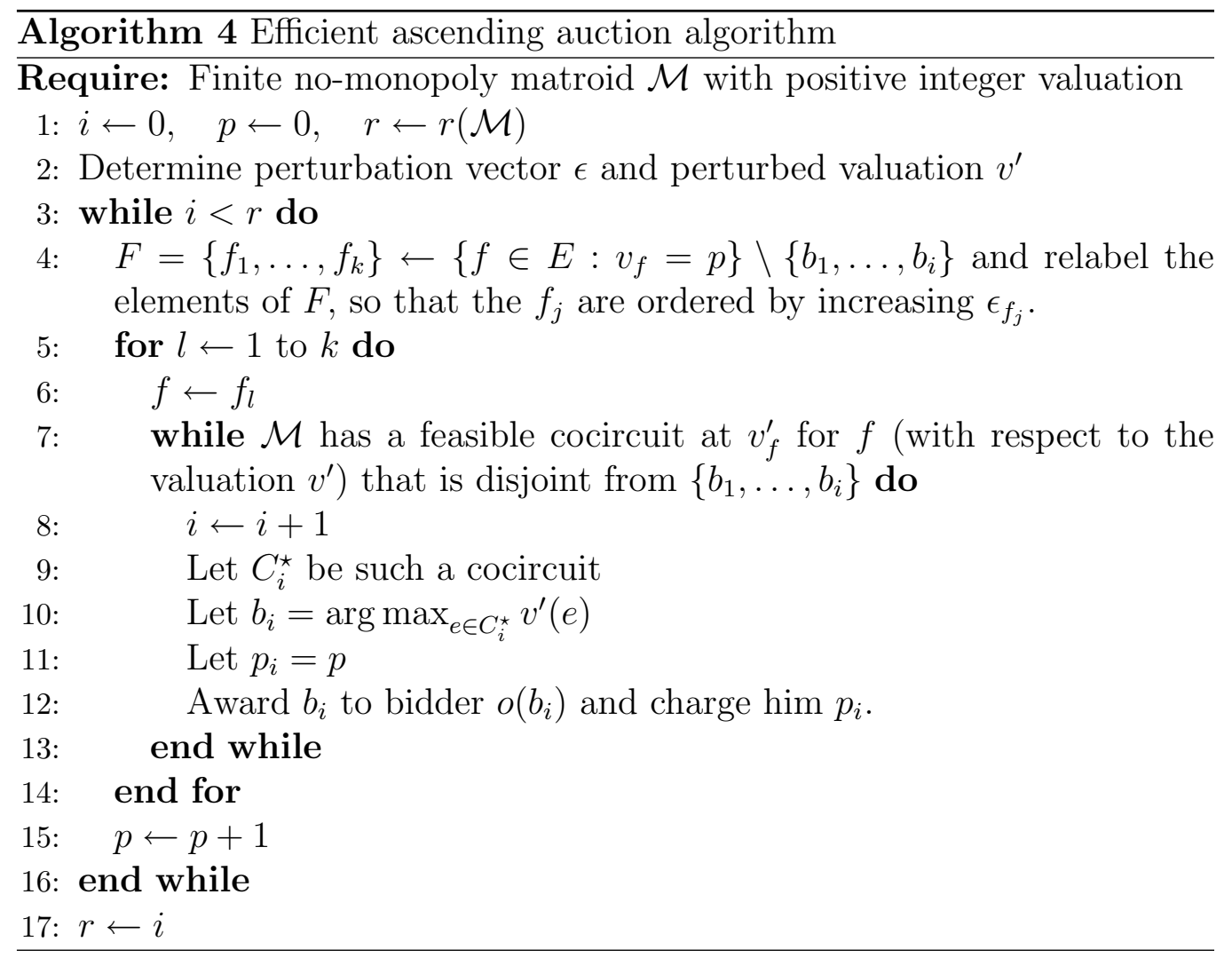

This makes the following theorem a direct consequence of the previous lemma.

Theorem 11. Algorithm \& applied to a finite, no-monopoly matroid $\mathcal{M}$ with positive, integer valuations determines an optimal basis, allocates it, and charges VCG-prices.

\subsection{An Ascending Auction Interpretation}

The algorithm described in the previous subsection is here rewritten as Auction 5 .

With Lemma 8, we see that deletion of non-chosen elements of value strictly less than $p$ does not change the outcome. Therefore, in Auction 5, 


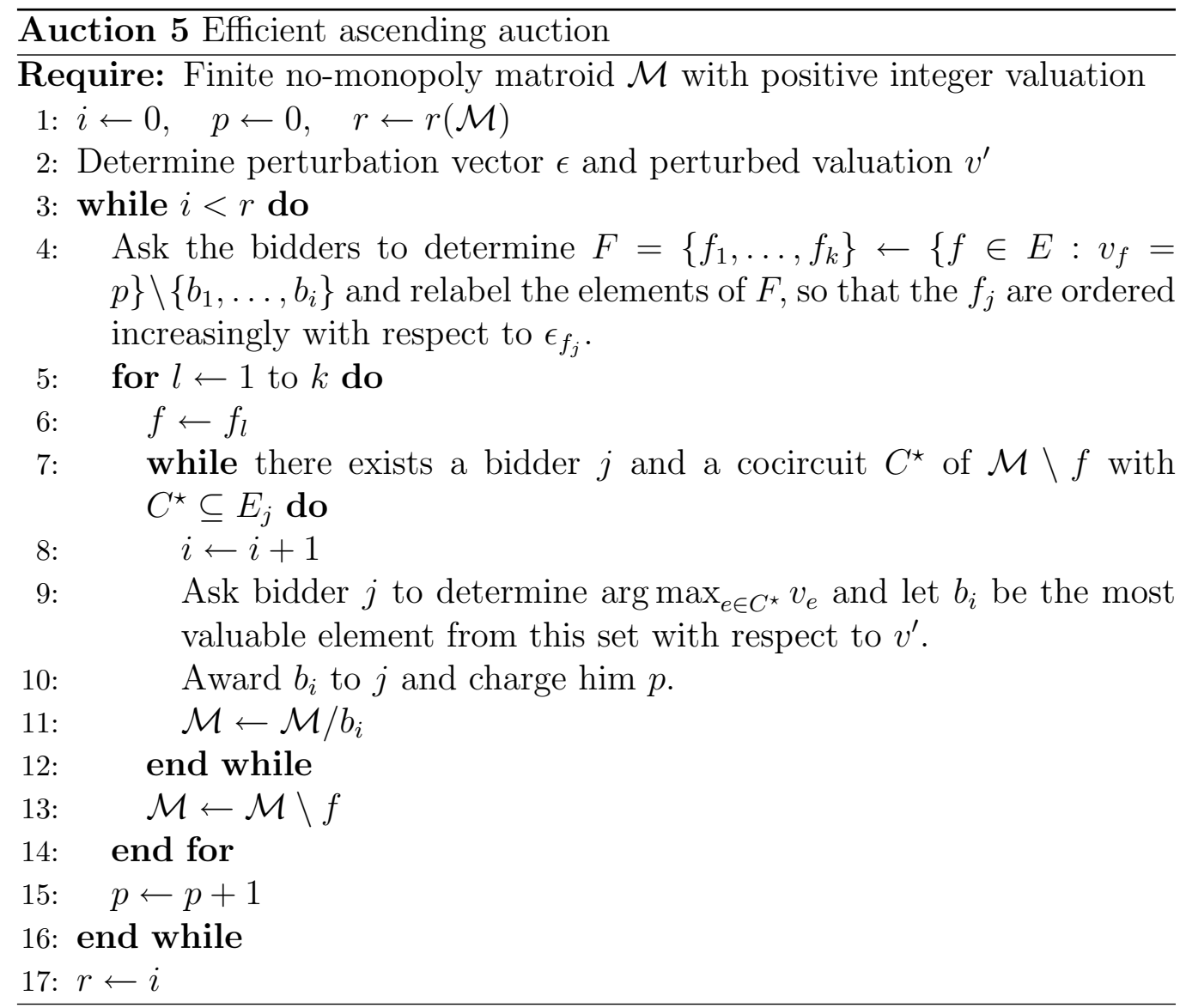

the elements that are not awarded and that are of value less than $p$ are discarded by deleting them from $\mathcal{M}$.

Lemma 12. If $\left(\left(C_{1}^{\star}, b_{1}\right),\left(C_{2}^{\star}, b_{2}\right), \ldots,\left(C_{r}^{\star}, b_{r}\right)\right)$ is suitable for $\mathcal{M}$, then $\left(\left(C_{2}^{\star}, b_{2}\right)\right.$, $\left.\ldots,\left(C_{r}^{\star}, b_{r}\right)\right)$ is suitable for $\mathcal{M} / b_{1}$. If $b_{1} \notin\left\{b_{2}, \ldots, b_{r}\right\}$, and $\left(\left(C_{2}^{\star}, b_{2}\right), \ldots\right.$, $\left.\left(C_{r}^{\star}, b_{r}\right)\right)$ is suitable for $\mathcal{M} / b_{1}$, and $C_{1}^{\star} \ni b_{1}$ is a cocircuit of $\mathcal{M}$ with $b_{1}=$ $\arg \max _{e \in C_{1}^{\star}} v_{e}$, then $\left(\left(C_{1}^{\star}, b_{1}\right),\left(C_{2}^{\star}, b_{2}\right), \ldots,\left(C_{r}^{\star}, b_{r}\right)\right)$ is suitable for $\mathcal{M}$.

Proof. Notice that cocircuits of $\mathcal{M}$ disjoint from $b_{1}$ are also cocircuits of $\mathcal{M} / b_{1}$ (dual of Proposition 20). Cocircuits of $\mathcal{M} / b_{1}$ are cocircuits of $\mathcal{M}$ (clear, by dualizing the statement). The lemma now follows.

By Lemma 12 it suffices to consider cocircuits of $\mathcal{M} /\left\{b_{1}, \ldots, b_{i}\right\}$ instead of all the cocircuits of $\mathcal{M}$ which avoid $\left\{b_{1}, \ldots, b_{i}\right\}$. 
Another observation is that whenever the deletion of a element $f$ would create a matroid violating the no-monopoly condition, then from each cocircuit witnessing this, one element is contracted; hence after the contractions and then the deletion, the no-monopoly condition holds again.

This yields the following theorem:

Theorem 13. For every finite matroid with positive integer valuation where no bidder has a monopoly, our Auction 5 determines an efficient allocation and charges Vickrey prices.

The proposed auction can be carried out in the following way. Start with the price set at zero. At this price, by assumption no bidder owns a monopoly. Increase the price. If any bidder indicates that he has an element of this value, then consider the element $f$ with smallest $\epsilon_{f}$ among them, check whether, after removing this element, another bidder $j$ owns a monopoly (i.e. $\mathcal{M} \backslash f$ has a cocircuit $C^{\star}$ contained in $E_{j}$; this means it is feasible, since $C^{\star}$ is feasible at some $p$ for $f$ with $v_{f}=p$ if the set $\left\{e \in C^{\star}: v_{e}>p\right\}$ is contained in some $E_{i}$ that avoids $f$ ). If this is the case, let bidder $j$ determine his best elements from $C^{\star}$ and award to him the unique element $e \in C^{\star}$ with maximum $v^{\prime}$-value and charge him the current price. Then contract $e$ and check for any other bidders that might own a cocircuit. Afterwards delete the unnecessary element $f$ from the matroid, increase $p$, and continue.

\subsection{Homogeneous Goods with Decreasing Marginal Val- ues}

For one application of our ascending matroid auction consider an auction where $k$ identical units must be auctioned off to $n$ bidders. Denote the (marginal) value that bidder $j$ assigns to consuming his $i^{\text {th }}$ unit by $v_{i}^{j}$. Here we consider (as Ausubel (2004) did) the case in which bidders have decreasing marginal valuations: $v_{i}^{j} \geq v_{i+1}^{j}$ for each $i \leq k-1$. Under this assumption, the problem of finding an efficient allocation can be formulated as the problem of finding a maximum weight basis.

First we recall some facts about uniform matroids. The uniform matroid 
$U_{k, l}$ for $k \leq l$ has a ground set with $l$ elements and has

$$
\begin{aligned}
\mathcal{I}\left(U_{k, l}\right) & =\{X \subseteq E:|X| \leq k\} \\
\mathcal{C}\left(U_{k, l}\right) & = \begin{cases}\emptyset & \text { if } k=l \\
\{X \subseteq E:|X|=k+1\} & \text { if } k<l .\end{cases}
\end{aligned}
$$

Regarding duality we see that $U_{k, l}^{\star}=U_{l-k, l}$ and deduce that the the cocircuits of $U_{k, l}$ are:

$$
\mathcal{C}^{\star}\left(U_{k, l}\right)=\{X \subseteq E:|X|=l-k+1\} .
$$

Let $T$ be a $t$-element subset of $E=E\left(U_{k, l}\right)$. Then

$$
\begin{gathered}
U_{k, l} / T \cong \begin{cases}U_{0, l-t} & \text { if } l \geq t \geq k, \\
U_{k-t, l-t} & \text { if } t<k ;\end{cases} \\
U_{k, l} \backslash T \cong \begin{cases}U_{l-t, l-t} & \text { if } l \geq t \geq l-k, \\
U_{k, l-t} & \text { if } t<l-k ;\end{cases}
\end{gathered}
$$

Now let $I=N \times K$ with $K:=\{1, \ldots, k\}$. Consider two integer vectors $\mathbf{l} \leq \mathbf{u}$, where (initally) $\mathbf{l}=\mathbf{0}$ and $\mathbf{u}=k \mathbf{1}$. Let $m$ always equal $\sum_{j=1}^{n}\left(u_{j}-l_{j}\right)$ (so initially $m=k n$ ). Now we consider the uniform matroid $\mathcal{U}_{k, m, \mathbf{l}, \mathbf{u}}$ with ground set

$$
E\left(\mathcal{U}_{k, m, \mathbf{l}, \mathbf{u}}\right)=\left\{(j, q) \in I: l_{j}<q \leq u_{j}\right\}
$$

and

$$
\mathcal{I}\left(\mathcal{U}_{k, m, \mathbf{l}, \mathbf{u}}\right)=\left\{F \subseteq E\left(\mathcal{U}_{k, m, \mathbf{l}, \mathbf{u}}\right):|F| \leq k\right\} .
$$

The value of $(j, q)$ is $v_{q}^{j}$; the interpretation is, that the elements $\{j\} \times K$ represent the $k$ units that bidder $j$ could get and their values correspond to his marginal values for the first up to the $k$-th unit. ${ }^{4}$ Clearly, the optimal bases of $\mathcal{U}_{k, m, \mathbf{l}, \mathbf{u}}$ corresponds to the best allocation of the $k$ units, because bidder $j$ gets, by decreasing marginal values, in an optimal basis the item $(j, q)$ only if he is awarded the items $(j, q-1), \ldots,(j, 1)$ that have higher marginal value. Hence the value of the optimal basis and of the best allocation agree too.

Now we need a couple of observations to understand the steps of Auction 5 applied to matroids of type $\mathcal{U}_{k, m, \mathbf{l}, \mathbf{u}}$. We will interpret elements $(j, 1), \ldots,\left(j, l_{j}\right)$

\footnotetext{
${ }^{4}$ In fact this construction has a deeper reason too. As a first attempt, one might start with a uniform matroid $\mathcal{U}_{k, k}$. But there is a problem. Different bidders want the same element. But as we noted before, adding parallel elements to achieve disjoint sets of interests is a way out. If this is done, a matroid isomorph to $\mathcal{U}_{k, k n}$ results.
} 
as already awarded elements and elements $\left(j, u_{j}+1\right), \ldots,(j, k)$ as elements in which the bidder $j$ is no longer interested (since their marginal value is lower than the current price). Therefore initializing $l_{j}=0, u_{j}=k$ is consistent with the inital situation that bidder $j$ is interested in all $k$ units and has not been awarded anything. Denote by $\mathbf{e}^{j}$ the $j$-th unit vector of $n$-space. We want to interpret the course of the matroid-auction, by keeping track of the vectors $\mathbf{l}, \mathbf{u}$.

For tie-breaking, we set $\epsilon_{j, q}=\delta^{j * k+q}$. This order ensures that the elements $(j, 1),(j, 2), \ldots,(j, k)$ are not only nonincreasing, but additionally strictly decreasing.

At any price $p$ during the run of the auction, there are no elements in the matroid with value strictly less than $p$. With $l=\left|E_{j} \cap F\right|$ holds (by our particular choice of tie-breaking) that the elements of $E_{j} \cap F$ (in increasing perturbation order) are $\left(j, u_{j}\right),\left(j, u_{j}-1\right), \ldots,\left(j, u_{j}-l+1\right)$. So for the element $f=f_{1}$ holds that $f=\left(o(f), u_{o(f)}\right)$. In line 13 of Auction 5 , where we set $\mathcal{M} \leftarrow \mathcal{M} \backslash f_{1}$ this reduces to replacing the matroid $\mathcal{U}_{k, m, \mathbf{l}, \mathbf{u}}$ by $\mathcal{U}_{k, m-1, \mathbf{l}, \mathbf{u}-\mathbf{e}^{o(f)}}$; since $f=\left(o(f), u_{o(f)}\right)$ belonged to the groundset of $\mathcal{U}_{k, m, \mathbf{l}, \mathbf{u}}$ we had that $l_{u(f}<u_{o(f)}$ it follows that $\mathbf{l} \leq \mathbf{u}-\mathbf{e}^{o(f)}$.

If in line 7 a cocircuit $C^{\star} \subseteq E_{j}$ in $\mathcal{U}_{k, m-1,1, \mathbf{u}-\mathbf{e}^{o(f)}}$ is selected then we know that $C^{\star}$ was not a cocircuit in $\mathcal{U}_{k, m, \mathbf{l}, \mathbf{u}}$ and by the no-monopoly condition bidder $o(e)$ possessed no cocircuit. But now he does. Notice that cocircuits of $\mathcal{U}_{k, m, \mathbf{l}, \mathbf{u}}$ are arbitrary subsets of size $m-k+1$ while the cocircuits of $\mathcal{U}_{k, m-1, \mathbf{l}, \mathbf{u}-\mathbf{e}^{o(f)}}$ are of size $m-k$. This requires that $u_{o(f)}-l_{o(f)}=m-k$ (if it were larger, then $o(f)$ had owned the cocircuit $C^{\star}$ already in $\mathcal{U}_{k, m, \mathbf{l}, \mathbf{u}}$; if it were

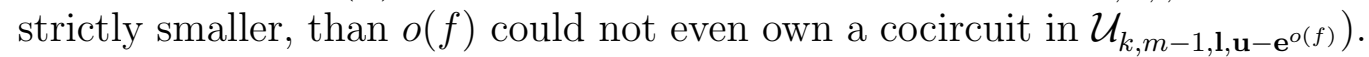

Given that $C^{\star}=\left\{\left(o(f), l_{o(f)+1}\right), \ldots,\left(o(f), u_{o(f)}\right)\right\}$ it is clear from the order and tie-breaking we use, that bidder $j$ 's answer to $\arg \max _{e \in C^{\star}} v_{e}$ is going to contain the element $\left(o(f), l_{o(f)+1}\right)$ which in turn is the highest $v^{\prime}$ valued element of bidder $o(f)$. Hence $b_{i}=\left(o(f), l_{o(f)+1}\right)$ has to be contracted in $\mathcal{M}$ resulting in a transition from $\mathcal{U}_{k, m, \mathbf{l}, \mathbf{u}}$ to $\mathcal{U}_{k-1, m-1, \mathbf{l}+\mathbf{e}^{o(f)}, \mathbf{u}}$.

Under the just presented specialization to uniform matroids $\mathcal{U}_{k, m, \mathbf{l}, \mathbf{u}}$ our Auction 5 reduces to the ascending auction for homogeneous goods with decreasing marginal values by Ausubel (2004).

If bidders do not have decreasing marginal valuations, then the efficient allocation problem is not equivalent to the problem of finding a maximum weight basis. For example, suppose that bidder 1 has a higher marginal value for his second object than for his first: $v_{1}^{1}<v_{2}^{1}$. In this case, (depending on the other bidders' valuations), a maximum weight basis may include the 
RCS: 1.43, 2005/05/19 01:10:53

element $(1,2)$, but not $(1,1)$. However, it is not feasible to give bidder 1 his marginal valuation for a second object without giving him a first object! The allocation problem in this case requires an additional constraint; an agent cannot receive an $i+1^{\text {st }}$ object without receiving an $i^{\text {th }}$ object. This side constraint destroys the matroid structure.

\section{Summary}

\section{References}

Ausubel, Lawrence M. (1997). An efficient ascending-bid auction for multiple objects. Manuscript, University of Maryland.

http://www. ausubel.com/auction-papers/

97wp-efficient-ascending-auction.pdf

Ausubel, Lawrence M. (2004). An efficient ascending-bid auction for multiple objects. The American Economic Review, 94(5):1452-1475. First published as the manuscript (Ausubel, 1997).

Cramton, Peter (1998). Ascending auctions. European Economic Review, 42(3-5):745-756.

Dawson, Jeremy E. (1980). Optimal matroid bases: An algorithm based on cocircuits. The Quarterly Journal of Mathematics. Oxford, 31(2):65-69.

de Vries, Sven, James Schummer, and Rakesh V. Vohra (2004). On ascending Vickrey auctions for heterogeneous objects. Manuscript.

Gul, Faruk and Ennio Stacchetti (1999). Walrasian equilibrium with gross substitutes. Journal of Economic Theory, 87(1):95-124.

Gul, Faruk and Ennio Stacchetti (2000). The English auction with differentiated commodities. Journal of Economic Theory, 92(1):66-95.

Oxley, James G. (1992). Matroid Theory. Oxford University Press, Oxford, GB. 
RCS: $1.43,2005 / 05 / 19$ 01:10:53

\section{A Summary of Matroid Facts}

We summarize here (following Oxley, 1992) terms and facts from matroid theory that will be used.

Sven's Comment: after convincing us, that everything is sound, we should really weed out most of this...

A matroid $\mathcal{M}$ is an ordered pair $(E, \mathcal{I})$ of a finite ground set $E$ and a set $\mathcal{I}$ of subsets of $E$ satisfying the axioms:

(I1) $\emptyset \in \mathcal{I}$.

(I2) If $I \in \mathcal{I}$ and $I^{\prime} \subseteq I$ then $I^{\prime} \in \mathcal{I}$.

(I3) If $I_{1}, I_{2} \in \mathcal{I}$ and $\left|I_{1}\right|<\left|I_{2}\right|$, then there is an element $e$ of $I_{2}-I_{1}$ such that $I_{1} \cup e \in \mathcal{I}$.

Subsets of $E$ that belong to $\mathcal{I}$ are called independent, all other sets are called dependent. By a (slight) abuse of notation, we will refer to the ground set of a matroid $\mathcal{M}$ as $E(\mathcal{M})$ and to the set of independent sets as $\mathcal{I}(\mathcal{M})$; similar abuses are to follow.

Minimal dependent sets of a matroid $\mathcal{M}$ are called circuits and circuits consisting of single element are called loops; the set of all circuits of a matroid is denoted with $\mathcal{C}$. The set of $\operatorname{circuits} \mathcal{C}(\mathcal{M})$ of a matroid is characterized by the following properties:

(C1) $\emptyset \notin \mathcal{C}$.

(C2) If $C_{1}, C_{2} \in \mathcal{C}$ and $C_{1} \subseteq C_{2}$ then $C_{1}=C_{2}$.

(C3) If $C_{1}, C_{2} \in \mathcal{C}, e \in C_{1} \cap C_{2}$, and $f \in C_{1} \backslash C_{2}$, then there exists a $C_{3} \in \mathcal{C}$ such that $f \in C_{3} \subseteq\left(C_{1} \cup C_{2}\right)-e$.

The Property (C3) is called strong circuit elimination; in fact (C3) can be replaced by the equivalent, though weaker looking, axiom (C3'):

(C3') If $C_{1}, C_{2} \in \mathcal{C}, C_{1} \neq C_{2}$, and $e \in C_{1} \cap C_{2}$, then there exists a $C_{3} \in \mathcal{C}$ such that $C_{3} \subseteq\left(C_{1} \cup C_{2}\right)-e$.

called weak circuit elimination.

An independent set that is maximal is called a basis of the matroid. The set of bases $\mathcal{B}$ of a matroid is characterized by the following two properties: 
RCS: 1.43, 2005/05/19 01:10:53

(B1) $\mathcal{B}$ is non-empty.

(B2) If $B_{1}, B_{2} \in \mathcal{B}$ and $x \in B_{1}-B_{2}$, then there is an element $y$ of $B_{2}-B_{1}$ such that $\left(B_{1}-x\right) \cup y \in \mathcal{B}$.

It is easy to see that all bases of a matroid have same cardinality.

The rank function $r: 2^{E} \mapsto \mathbb{N}_{0}$ of a matroid $\mathcal{M}$, assigns to each $X \subseteq E$ the size of the largest independent subset of $X$. This is denoted $r(X)$. The rank function $r$ satisfies the properties:

(R1) If $X \subseteq E$, then $0 \leq r(X) \leq|X|$.

(R2) If $X \subseteq Y \subseteq E$, then $r(X) \leq r(Y)$.

(R3) If $X$ and $Y$ are subsets of $E$, then

$$
r(X \cup Y)+r(X \cap Y) \leq r(X)+r(Y) .
$$

Notice, that for a circuit $C$ of $\mathcal{M}, r(C)=|C|-1$.

Consider a matroid $\mathcal{M}=(E, \mathcal{I})$ and some $X \subseteq E$. Let $\mathcal{I} \mid X:=\{I \subseteq$ $X: I \in \mathcal{I}\}$. It is easy to see that $\mathcal{M} \mid X:=(X, \mathcal{I} \mid X)$ is a matroid, called the restriction of $\mathcal{M}$ to $X$, or the deletion of $E-X$ from $\mathcal{M}$ denoted by $\mathcal{M} \backslash(E-X)$. As $\mathcal{M} \mid X$ is a matroid, all of its bases are equicardinal.

Proposition 14. Let $\mathcal{M}$ be a matroid and $\mathcal{B}^{*}(\mathcal{M}):=\{E(\mathcal{M})-B: B \in$ $\mathcal{B}(\mathcal{M})\}$. Then $\mathcal{B}^{*}(\mathcal{M})$ is the set of bases of a matroid on $E(\mathcal{M})$.

The matroid with ground set $E(\mathcal{M})$ and bases described in the previous proposition is called the dual matroid of $\mathcal{M}$ and is denoted by $\mathcal{M}^{\star}$. Independent sets of $\mathcal{M}^{\star}$ are called coindependent sets of $\mathcal{M}$; circuits and loops of $\mathcal{M}^{\star}$ are called cocircuits and coloops of $\mathcal{M}$; etc. Notice that $\mathcal{M}=\mathcal{M}^{\star \star}$.

Proposition 15 (Oxley, 1992, Prop. 2.1.9). For all subsets $X$ of the ground set $E$ of a matroid $\mathcal{M}$,

$$
r^{\star}(X)=|X|-r(\mathcal{M})+r(E-X) .
$$

Proposition 16. If $C^{\star}$ is a cocircuit of the matroid $\mathcal{M}$ then

$$
r\left(\mathcal{M}-C^{\star}\right)=r(\mathcal{M})-1 .
$$


RCS: 1.43, 2005/05/19 01:10:53

Proof. Take a basis $B^{\prime}$ of $\mathcal{M} \backslash C^{\star}$. As it is independent in $\mathcal{M}$ it can be augmented to a basis $B \supseteq B^{\prime}$ of $\mathcal{M}$. Now $E-B$ is a cobasis; so it is coindependent and for its intersection with $C^{\star}$ holds: $\left|(E-B) \cap C^{\star}\right| \leq\left|C^{\star}\right|-1$. Hence $\left|B \cap C^{\star}\right| \geq 1$ and $|B|-1 \geq\left|B^{\prime}\right|$. This implies $r(\mathcal{M})-1 \geq r\left(\mathcal{M}-C^{\star}\right)$.

Conversely, notice that the set $I^{\star}=C^{\star}-e$ (for any $e \in C^{\star}$ ) is coindependent. Hence it can be augmented to a cobasis $B^{\star}$; furthermore, as $C^{\star}$ is a cocircut and $C^{\star}-e \subseteq B^{\star}$ it follows $e \notin B^{\star}$. For $B:=E-B^{\star}$ follows that $B \cap C^{\star}=\{e\}$ which implies, that $\left|B-C^{\star}\right|=|B|-1=r(\mathcal{M})-1$. Hence $r(\mathcal{M})-1 \leq r\left(\mathcal{M}-C^{\star}\right)$.

For a matroid $\mathcal{M}$ and a subset $T$ of its ground set $E$ we let $\mathcal{M} / T:=$ $\left(\mathcal{M}^{\star} \backslash T\right)^{\star}$ denote the contraction of $T$ from $\mathcal{M} . \mathcal{M} / T$ has ground set $E-T$.

Proposition 17 (Oxley, 1992, Prop. 3.1.4). If $T \subseteq E$, then, for all $X \subseteq$ $E-T$

$$
r_{\mathcal{M} / T}(X)=r_{\mathcal{M}}(X \cup T)-r_{\mathcal{M}}(T) .
$$

Any matroid $\mathcal{M}^{\prime}$ that can be produced by a sequence of contractions and deletions from the matroid $\mathcal{M}$ is called a minor of $\mathcal{M}$.

Proposition 18 (Oxley, 1992, Prop. 3.1.8). Suppose that $B_{T}$ is a basis for $\mathcal{M} \mid T$. Then $\mathcal{I}(\mathcal{M} / T)=\left\{I \subseteq E-T: I \cup B_{T} \in \mathcal{I}(\mathcal{M})\right\}$.

Proposition 19 (Oxley, 1992, Prop. 3.1.11). The circuits of $\mathcal{M} / T$ consist of the minimal non-empty members of $\{C-T: C \in \mathcal{C}(\mathcal{M})\}$.

Proposition 20 (Oxley, 1992, 3.1.13).

$$
\mathcal{C}(\mathcal{M} \backslash T)=\{C \subseteq E-T: C \in \mathcal{C}(\mathcal{M})\} .
$$

Proposition 21 (Oxley, 1992, Cor. 3.1.25). $\mathcal{M} \backslash e=\mathcal{M} / e$ if and only if $e$ is a loop or coloop of $M$.

Sven's Comment: the following nonstandard result is used later; please check carefully...

Proposition 22 (Oxley, 1992, Exc. 2, Sec 3.1). Let $C$ be a circuit of the matroid $\mathcal{M}$ and $e \in E(\mathcal{M})$ :

(i) If $e \notin C$ then $C$ is a union of circuits of $\mathcal{M} / e$.

(ii) If $e \in C$ and $\{e\}$ is a not a loop of $\mathcal{M}$, then $C-e$ is a circuit of $\mathcal{M} / e$. 
Proof. For (i): If $e$ is a loop then $C$ is a circuit of $M \backslash e$ but $\mathcal{M} \backslash e=\mathcal{M} / e$ so $C$ is a circuit of $\mathcal{M} / e$ thereby validating the claim. So we assume now that $e$ is not a loop.

If $C$ is a loop of $\mathcal{M}$, then, as $C \cup\{e\}$ is dependent in $\mathcal{M}$, the set $C$ is dependent in $\mathcal{M} / e$. As it contains only one element, clearly $C$ is a circuit of $\mathcal{M} /$ e. So we assume now, that $C$ contains at least two elements.

Now consider an element $f \in C$. Notice $C \backslash f$ is independent in $\mathcal{M}$; as $C$ is dependent in $\mathcal{M}$ so is $C \cup e$, therefore $C$ is dependent in $\mathcal{M} / e$.

Case 1: If $(C \cup e) \backslash f$ is independent in $\mathcal{M}$, then $C \backslash f$ is independent in $\mathcal{M} / e$. But now we have that $C$ is dependent and $C \backslash f$ is independent in $\mathcal{M} / e$; hence there has to be a circuit in $\mathcal{M} / e$ contained in $C$ thru $f$.

Case 2: If on the other hand $(C \cup e) \backslash f$ is dependent in $\mathcal{M}$, then it contains a circuit $D$ thru $e$. As $\{e\}$ is not a loop, $|D| \geq 2$; let $g$ be an element of $D \backslash e$; notice $g \in C$. By strong circuit elimination, there is a circuit $D^{\prime} \subseteq(C \cup D) \backslash g$ containing $e$. So $D^{\prime} \subseteq(C \cup e) \backslash g$ and $D^{\prime} \backslash\{e, g\} \subsetneq C$ is dependent in $\mathcal{M} / e$.

But $C \backslash f$ is independent, so $C \cup e \backslash f$ contains a single circuit $D$ with $g \in D$ and $(C \cup e) \backslash\{f, g\}$ is independent in $\mathcal{M}$. Hence $C \backslash\{f, g\}$ is independent in $\mathcal{M} / e$. On comparing (in $\mathcal{M} / e$ ) the independent set $C \backslash\{f, g\}$ with the dependent set $D^{\prime} \backslash\{e, g\} \subsetneq C$ notice that $\left(D^{\prime} \backslash\{e, g\}\right) \backslash(C \backslash\{f, g\}) \subseteq\{f\}$. This shows that the circuit $D^{\prime} \backslash\{e, g\}$ contains $f$ and is contained in $C$.

For (ii): As $\{e\}$ is not a loop, $\{e\} \neq C$. As $e \cup(C-e)$ is dependent in $\mathcal{M}$, the set $C-e \neq \emptyset$ is dependent in $\mathcal{M} / e$. As for any subset $I \subsetneq(C-e)$ the set $I+e$ is independent in $\mathcal{M}$, the set $I$ is independent in $\mathcal{M} / e$. So in fact, $(C-e)$ is minimally dependent in $\mathcal{M} / e$.

Since $\mathcal{M}^{\star} / e=(\mathcal{M} \backslash e)^{\star}$ we deduce:

Proposition 23. Let $C^{\star}$ be a cocircuit of the matroid $\mathcal{M}$ and $e \in E(\mathcal{M})$ :

(i) If $e \notin C^{\star}$ then $C^{\star}$ is a union of cocircuits of $\mathcal{M} \backslash e$.

(ii) If $e \in C^{\star}$ and $\{e\}$ is a not a coloop of $\mathcal{M}$, then $C^{\star}-e$ is a cocircuit of $\mathcal{M} \backslash e$.

Corollary 24. Let $C^{\star}$ be a cocircuit of the matroid $\mathcal{M}$ and $T \subseteq E(\mathcal{M})$, then $C^{\star}-T$ is the union of cocircuits of $\mathcal{M} / T$. 